\title{
BMJ Open Textured shoe insoles to improve balance performance in adults with diabetic peripheral neuropathy: study protocol for a randomised controlled trial
}

\author{
Anna L Hatton, ${ }^{1}$ Elise M Gane, ${ }^{1}$ Jayishni N Maharaj, ${ }^{2}$ Joshua Burns, ${ }^{3}$ \\ Joanne Paton, ${ }^{4}$ Graham Kerr, ${ }^{5}$ Keith Rome ${ }^{6}$
}

To cite: Hatton AL, Gane EM, Maharaj JN, et al. Textured shoe insoles to improve balance performance in adults with diabetic peripheral neuropathy: study protocol for a randomised controlled trial. BMJ Open 2019;9:e026240. doi:10.1136/ bmjopen-2018-026240

- Prepublication history for this paper is available online. To view these files, please visit the journal online (http://dx.doi org/10.1136/bmjopen-2018026240).

Received 23 August 2018 Revised 18 April 2019 Accepted 11 June 2019
Check for updates

(C) Author(s) (or their employer(s)) 2019. Re-use permitted under CC BY-NC. No commercial re-use. See rights and permissions. Published by BMJ.

For numbered affiliations see end of article.

Correspondence to

Dr Anna L Hatton;

a.hatton1@uq.edu.au

\section{ABSTRACT}

Introduction Peripheral neuropathy is a major risk factor for falls in adults with diabetes. Innovative footwear devices which artificially manipulate the sensory environment at the feet, such as textured shoe insoles, are emerging as an attractive option to mitigate balance and walking problems in neuropathic populations. This study aims to explore whether wearing textured insoles for 4 weeks alters balance performance in adults with diabetic peripheral neuropathy.

Methods and analysis A prospective, single-blinded randomised controlled trial with parallel groups will be conducted on 70 adults with diabetic peripheral neuropathy. Adults with a diagnosis of peripheral neuropathy (secondary to type 2 diabetes), aged $\geq 18$ years, ambulant over $20 \mathrm{~m}$ (with/without an assistive device), will be recruited. Participants will be randomised to receive a textured insole $(n=35)$ or smooth insole $(n=35)$, to be worn for 4 weeks. During baseline and post intervention assessments, standing balance (foam/ firm surface; eyes open/closed) and walking tasks will be completed barefoot, wearing standard shoes only, and two different insoles (smooth, textured). The primary outcome measure will be centre of pressure (CoP) velocity, with higher values indicating poorer balance. Secondary outcome measures include walking quality (gait velocity, base of support, stride length and doublelimb support time), physical activity levels, foot sensation (light-touch pressure, vibration) and proprioception (ankle joint position sense), and other balance parameters ( $\mathrm{CoP}$ path length, anteroposterior and mediolateral excursion). Patient-reported outcomes will be completed evaluating foot health, frequency of falls and fear of falling. Data will be analysed using a repeated measures mixed models approach (including covariates) to establish any differences between-groups, for all outcome measures, over the intervention period.

Ethics and dissemination Ethical approval has been obtained from the institutional Human Research Ethics Committee (\#2017000098). Findings will be disseminated at national and international conferences, through peerreviewed journals, workshops and social media.

Trial registration number ACTRN12617000543381; Preresults.

\section{Strengths and limitations of this study}

- This study will be among the first to explore whether novel shoe insoles can provide substitute sensory input to the soles of the feet, to help mitigate balance problems in adults with diabetic peripheral neuropathy.

- If the trial demonstrates a positive effect of textured insoles, the findings will be used to inform the development of innovative, affordable, non-invasive neuropathic treatment devices, which target diabetic foot sensory complications that contribute to falls.

- This study is limited to assessing the effects of wearing textured insoles for 4 weeks on balance, walking, physical activity and patient-reported outcomes: exploration of long-term wear, whereby any beneficial effects may accrue over time, will be tested in future trials.

\section{INTRODUCTION}

Peripheral neuropathy affects between 22\% and $51 \%$ of people with diabetes, ${ }^{12}$ and is a major risk factor for falls, reported to be present in the lower limbs of up to $86 \%$ of older diabetic fallers. ${ }^{3}$ Nerve damage can severely disrupt the quality of sensory information transmitted from the feet to the brain, which provides vital cues about the supporting surface and position of body segments, to help older people remain upright. Similarly, balance impairments can also be observed in younger adults with diabetic peripheral neuropathy, placing them too at risk of sustaining falls-related injuries, with potential for deterioration with increasing age. ${ }^{4}$ Loss of cutaneous sensory information from the foot sole is reported to be a critical factor contributing to walking instability, poor balance ${ }^{5}$ and falls, ${ }^{6}$ in people with diabetic peripheral neuropathy. 
Foot care is a standard practice in the management of diabetic populations, with shoe insoles routinely prescribed by healthcare professionals to help prevent foot ulceration, ${ }^{78}$ or provide pain relief, by way of redistributing plantar pressures. The effect of traditional footwear devices on balance performance in people with diabetic peripheral neuropathy has received little attention, with inconclusive evidence both supporting ${ }^{9} 10$ and refuting, ${ }^{11}$ the efficacy of shoe insoles. In contrast, lower limb strengthening exercises demonstrate stronger evidence as an effective balance intervention for people with diabetic neuropathy, ${ }^{9}$ which may also have the capacity to improve cutaneous reinnervation. ${ }^{12}$ However, innovative footwear devices which artificially manipulate the sensory environment at the feet are emerging as an attractive treatment option, which may compliment current exercise strategies to help mitigate balance and walking problems and reduce the risk of falling in adults with diabetes. ${ }^{13-15}$ Textured shoe insoles, designed to stimulate cutaneous receptors on the soles of the feet and provide enhanced sensory feedback, have been shown to improve lateral (sideways) standing balance in healthy older adults, ${ }^{13}$ and those with Parkinson's disease. ${ }^{16}$ This is a clinically important finding, as lateral balance is reported to be a strong predictor of falls. ${ }^{17}{ }^{18}$ Furthermore, in older fallers, ${ }^{14}$ and adults with multiple sclerosis, ${ }^{19}$ wearing textured insoles have also brought about changes in spatiotemporal gait measures, synonymous with more confident and stable walking patterns.

The potential therapeutic effects of textured insoles in people with pathological sensory loss largely remain unknown, due to the exclusion of this population from previous research strategies. However, a systematic review by Paton $e t a l^{20}$ revealed that people with pathological loss of foot sensation may benefit most from wearing footwear devices which augment the plantar sensory environment. There is limited evidence exploring the concept of lower limb sensory devices for adults with diabetic peripheral neuropathy. ${ }^{10} 2122$ One study applied tactile stimulation to the calf, therefore bypassing critical foot regions showing neuropathic damage. ${ }^{22}$ A further study explored insoles comprising in-built, battery-powered, vibrating mecht$\operatorname{rodes}^{10}$ : a design feature which is impractical, complex to integrate into everyday footwear and expensive as a neuropathic treatment. Paton et $a l^{21}$ provided new evidence to demonstrate that wearing textured insoles may heighten reliance on, and responsiveness to, plantar sensory inputs even in the presence of severe diabetic peripheral neuropathy. Further, compelling evidence by Wegener has demonstrated that wearing sensorimotor foot orthoses can alter ankle, knee and hip motion during walking, which may translate to increased gait stability, in adults with inherited neuropathy (Charcot-Marie-Tooth disease) ${ }^{23}$ This area urgently requires investigation in the real world context to understand the clinical impact in people with diabetic peripheral neuropathy. Textured insoles may offer a new rehabilitative approach, which promotes self-management by the user, to improve balance and walking, and reduce the risk of falling, in adults with diabetes.

\section{Study aims}

This study protocol describes a randomised controlled trial of textured insoles, worn over a 4-week period, in adults with diabetic peripheral neuropathy. The primary aim of the study is to determine if wearing textured insoles for 4 weeks alters measures of standing balance ability (centre of pressure ( $\mathrm{CoP})$ velocity), under different conditions where visual information and the supporting surface are manipulated. Secondary aims are to determine if shortterm wear of textured insoles leads to changes in walking quality (spatiotemporal gait parameters: gait velocity, base of support, stride length and double-limb support time), foot sensation (light touch pressure, vibration, ankle joint proprioception), physical activity levels using wearable sensors, other standing balance parameters (CoP path length, anteroposterior and mediolateral excursion) and patient-reported outcomes (foot-health specific quality of life, frequency of self-reported falls and fear of falling).

\section{METHODS AND ANALYSIS \\ Study design}

A prospective, parallel group, single-blinded, randomised controlled trial with 70 community-dwelling adults, living with diabetic peripheral neuropathy will be conducted and reported, conforming to the Standard Protocol Items for Randomised Trials statement (figure 1). ${ }^{24}$

\section{Location and setting}

All assessments will be conducted within the Centre for Neurorehabilitation, Ageing and Balance Research gait laboratory at The University of Queensland (UQ), Australia.

\section{Participants}

Recruitment of participants commenced on 14 August 2017 and remains ongoing. Community-dwelling men and women with a clinical diagnosis of diabetic peripheral neuropathy secondary to type 2 diabetes will be identified through a pool of sampling frames including Diabetes Australia, Diabetes Queensland and local community organisations across the Brisbane, Gold Coast, Sunshine Coast and Logan regions, via mainstream media advertisements, including social media (eg, Facebook). Participants will also be identified through research volunteer databases maintained by the Centre for Neurorehabilitation, Ageing and Balance Research at UQ and Movement Neuroscience Programme at Queensland University of Technology (QUT); UQ and QUT Health Clinics, whereby recruitment processes will be coordinated by clinical staff to maintain patient confidentiality.

Participants will be permitted to take part in the trial if they meet the following criteria: aged $\geq 18$ years; clinical diagnosis of diabetic peripheral neuropathy secondary to type 2 diabetes; ambulant over $20 \mathrm{~m}$ (with or without 


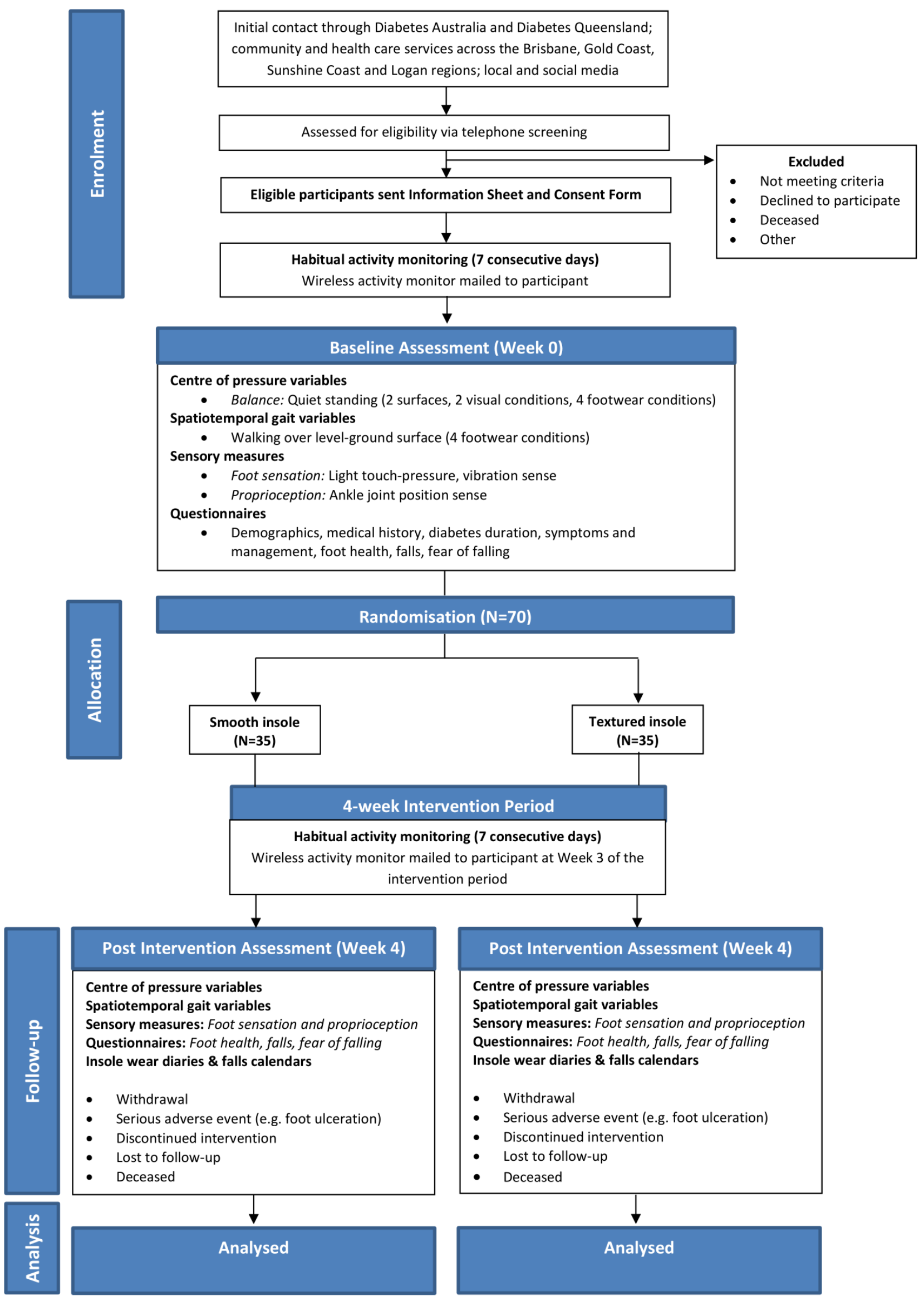

Figure 1 Trial design. 
the use of an assistive device, eg, single cane); willing to wear shoe insoles for 4 weeks; and have adequate English communication skills to provide informed consent and comprehend the study procedures. A diagnosis of diabetic peripheral neuropathy will be determined using one of two criteria. First, participants will be asked to provide details of their diagnosis of type 2 diabetes and peripheral neuropathy, previously given by a health professional. Second, participants who have a diagnosis of type 2 diabetes, but not of peripheral neuropathy, yet self-report significant alterations in lower limb sensation synonymous with neuropathy, will be screened using the Diabetic Neuropathy Symptom Score to determine eligibility. ${ }^{25}$ This tool comprises four items addressing the nature and frequency of neuropathic symptoms (experienced across both lower limbs collectively) including (1) unsteadiness in walking; (2) burning, aching pain or tenderness in the legs or feet; (3) prickling sensations in the legs or feet and; (4) numbness in the legs or feet, experienced in the last 2 weeks. The presence of one symptom is scored as 1 point, and a total score of $1-4$ points indicates the presence of peripheral neuropathy. This tool has comparable sensitivity (79\%) and higher specificity (78\%) compared with physical examination techniques (monofilaments: $81 \%$ sensitivity, $56 \%$ specificity; vibration perception threshold: $81 \%$ sensitivity, $58 \%$ specificity), ${ }^{25}$ and is recommended by Diabetes Australia and The Royal Australian College of General Practitioners, ${ }^{26}$ as a method for confirming a diagnosis of peripheral neuropathy. Exclusion criteria are current foot ulceration or other lower limb injury; current use of over-the-counter or custom-made foot orthoses or shoe insoles; previous history of lower limb or partial foot amputation, Charcot Arthropathy, other neurological diseases or cardiorespiratory conditions that limit ambulation; unstable psychiatric condition (defined by a qualified health professional, eg, general practitioner) or cognitive impairment (Short Form Mini-Mental State Examination Score <24) ${ }^{27}$ All participants will initially be screened via telephone interview, prior to attending faceto-face assessments.

\section{Randomisation and blinding}

Participants will be randomised to either the textured (intervention) insole or smooth (control) insole group. The concealed, computer-generated block randomisation schedule will be managed by an independent offsite investigator who is not involved in the enrolment or assessment of participants. Consecutively numbered, opaque envelopes, containing group allocation (in a 1:1 ratio), will be opened after the baseline assessment by the study podiatrist (JNM) who is solely responsible for fitting and issuing the insoles. All other investigators and research assistants, responsible for enroling and assessing participants, will remain blind to the group allocation. Following baseline assessment, the first and second research assistants will leave the gait laboratory to ensure blinding to the insole condition. The podiatrist will then customise and fit the

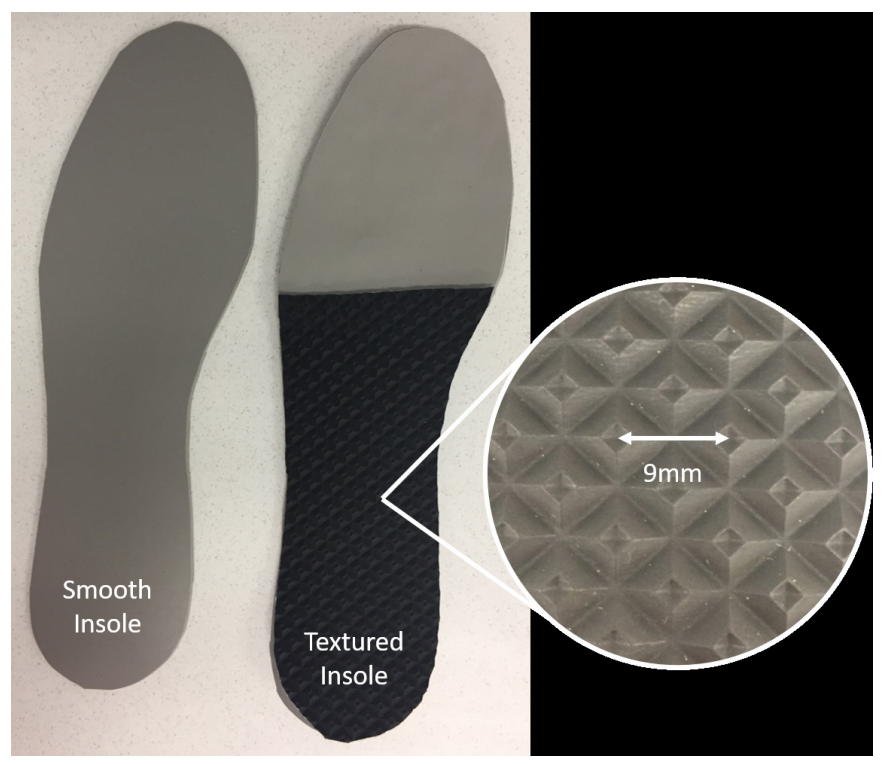

Figure 2 Textured and smooth shoe insoles.

participant with their allocated insole, and provide advice regarding the frequency of wear, completion of insole wear and falls diaries. Participants will be instructed not to divulge their insole allocation. The second research assistant will be responsible for maintaining the within-session insole condition randomisation schedule and coding, and for fitting the different insoles and shoes during assessments. During baseline assessment and post intervention assessment, the first research assistant will remain blinded to the order of testing of insole conditions. Due to the nature of the insole material being used, it is not possible to blind participants to their allocated group (ie, those in the intervention group may perceive the textured surface against their foot or visibly see the geometric pattern on the upper surface of the insole), therefore, the identity of the intervention insole will not be concealed. Participants will not be informed which insole condition is intended to provide enhanced plantar sensory feedback or is under investigation for its capacity to alter balance performance. Debriefing of participants will occur on completion of the trial. Additionally, participant coding will not refer to group allocation.

\section{Intervention}

Two different shoe insoles will be investigated in this randomised controlled trial: textured (intervention) insoles and smooth (control) insoles (figure 2). Importantly, both insoles have been implemented in previous research strategies, ${ }^{21}$ and incorporate materials typically used for insoles prescribed to people with diabetic peripheral neuropathy. The insoles will be custom-made to each participant's foot and shoe length. The textured insole will be constructed from $3.2 \mathrm{~mm}$ Poron (Poron 4000, Algeos, VIC, Australia), covered with $100 \%$ polyvinyl chloride (PVC) material which has raised pyramidal nodules (Techno PVC covering, Black Tech, Algeos, VIC, Australia) with centre-to-centre distances of $9 \mathrm{~mm}$. The 
PVC material will be adhered to the Poron base using contact adhesive (Selley's Kwik Grip Crystal Clear, DeluxGroup, VIC, Australia). The textured zone will extend from the heel to the proximal aspect of the first-fifth metatarsal heads, to avoid excessive friction at the forefoot. ${ }^{28}$ To minimise increased pressure that could lead to skin damage at the exposed edge of the textured material, a forefoot extension ( $1 \mathrm{~mm}$, Poron 4000) will be adhered to the Poron base, extending proximal to the metatarsal heads to the distal end of the insole. This forefoot extension will be bevelled to create a smooth transition from the textured zone. The length of the textured zone and forefoot extension will be customised to each participant, by the podiatrist (JNM) at the baseline assessment. The smooth insole will only comprise the $3.2 \mathrm{~mm}$ Poron (with no PVC top cover). All edges of the textured and control insoles will be smoothed and shaped to fit the intended footwear using an orthopaedic grinder prior to dispense. Participants will be instructed to wear their allocated insoles, in their own shoes, as much as possible (when indoors and outdoors): wear time will be gradually increased. Assessments of balance and gait will be conducted with participants wearing standard shoes (following a $5 \mathrm{~min}$ familiarisation period) used in our previous work, ${ }^{16} 29$ comprising a basic construct rubbersoled shankless shoe with a soft canvas upper (Volley International Canvas, Volley, China), into which the insoles will be inserted. This standardisation will control for any possible insole/shoe interactions which could influence the findings. The shoes will be available in a range of sizes. Participants in both groups will be advised to continue with their usual care, and that athletic socks are the thickest permitted to be worn, over the intervention period.

\section{Primary outcome measure \\ CoP movement}

The primary outcome measure will be CoP velocity $\left(\mathrm{mm} . \mathrm{s}^{-1}\right)$ during bilateral standing, providing a measure of unperturbed balance control. CoP velocity represents the speed at which the body's centre of mass is moving, with a higher value indicating more rapid and potentially unstable movement. ${ }^{30}$ Participants will stand on an AMTI force platform (sampling rate $1000 \mathrm{~Hz}$ ), using standardised foot positioning (heels placed 1/10th participants height apart and angled to $\left.14^{\circ}\right),{ }^{31}$ with arms hanging by their sides, ${ }^{13}$ for $30 \mathrm{~s}$. Standing balance will be performed on a firm and foam $(50 \times 41 \times 15 \mathrm{~cm}$ foam block, placed directly on top of the force platform) surface, with eyes open and eyes closed, each while barefoot, wearing standard shoes only, and when wearing two different insoles (textured, smooth) within standard shoes (three trials per condition). The effects of texture may be more clearly identified during challenging tests of balance, which include the use of unstable surfaces such as foam. ${ }^{15}{ }^{16}$ During eyes open trials, participants will be instructed to look straight ahead at a black, circular visual target $(15 \mathrm{~cm}$ diameter), located $2.5 \mathrm{~m}$ from the centre of the force platform and adjusted to each participants' eye level. The test sequence (shoe condition, surface and vision) will be randomised. Where a participant overbalances or moves out of the standardised positioning, the trial will be discarded and repeated.

\section{Secondary outcome measures}

Spatiotemporal gait parameters

Spatiotemporal gait parameters will be evaluated as a measure of walking quality, including gait velocity, mediolateral base of support, stride length and double-limb support time (and step-to-step variability of each parameter). Level-ground gait performance will be evaluated by completing a $10 \mathrm{~m}$ walk over an instrumented, vinyl walkway (GAITRite CIR Systems, USA). The GAITRite has been shown to have high reliability. ${ }^{32} 33$ A start and finish line will be marked on the floor $0.63 \mathrm{~m}$ in front and $0.63 \mathrm{~m}$ behind the walkway to allow participants to accelerate and decelerate outside the walkway. ${ }^{32}$ Five walking trials will be completed at participants' comfortable, self-selected pace, while barefoot, wearing standard shoes only and wearing two different shoe insoles (textured, smooth) within standard shoes. Participants will be instructed to look straight ahead while walking. The test sequence (footwear condition) will be randomised.

\section{Physical activity}

Habitual physical activity levels will be measured over seven consecutive days, using a small wireless activity monitor (ActivPAL, Glasgow, Scotland), on two separate occasions: 1 week prior to the administration of insoles and during the final week of the 4-week intervention period. ${ }^{34}$ Measures of physical activity (per hour) will include time spent sitting, standing, stepping (minutes); number of sit-to-stand and stand-to-sit transitions; number of steps taken and; energy expenditure (metabolic equivalent). Previous evidence has demonstrated that the ActivPAL is a reliable and valid device for measuring posture and movement in adults during daily physical activities,${ }^{35}$ including walking. ${ }^{36}$ The increasing use of accelerometry in people with peripheral neuropathy is attributed to its ability to allow monitoring of changes in walking impairments with disease progression. ${ }^{37} 38$ Activity data provide the ultimate measure of participation as it pertains to gait, so provides a vital measure to determine if textured insoles impact on daily activities.

\section{Foot sensation and proprioception}

Lower limb somatosensory function will be assessed bilaterally. Light touch-pressure sense will be determined by recording the smallest monofilament that the participant can perceive at five locations on each foot. ${ }^{39}$ Semmes-Weinstein monofilaments (1.65-6.65 gauges) will be used to determine tactile sensibility at the hallux, first and fifth metatarsal head, heel and dorsal foot. ${ }^{39} 40$ Monofilaments will be applied on three occasions per site, perpendicular to the skin for $1.5 \mathrm{~s}$, and the smallest perceived ( $\geq 2 / 3$ applications) indicating the participant's 
light-touch pressure sense threshold. Vibration perception will be assessed using a neurothesiometer (Briggate Medical Company, VIC, Australia) (frequency $100 \mathrm{~Hz}$, amplitude $0-50 \mathrm{~V}$ ), at the hallux and first metatarsophalangeal joint. Starting at $0 \mathrm{~V}$, the vibration will be gradually increased until the participant indicates they can feel the vibration (normal: $\leq 25 \mathrm{~V}$ ). ${ }^{41}$ The average of three readings will be calculated, per site. Foot position awareness (proprioception) will be assessed using the ankle joint angle reproduction test, ${ }^{42}$ to help determine whether textured insoles improve dorsiflexion and plantarflexion perception, which may translate to improved toe clearance during walking. The participant's ankle joint will be passively set (by the investigator) to three angles in plantarflexion and dorsiflexion directions, relative to a neutral foot position. To minimise extraneous cues and psychological processes that could influence the findings, the foot will be positioned at a variable time and trajectory. The participant will be asked to reposition the ankle joint at the target angle by moving only the foot segment. Accuracy in joint positioning will be determined by measuring the difference between the target and actual angles using an internet-based goniometer, which has high inter-rater (ICC2, $1=0.96$ to $>0.99$ ) and intra-rater (ICC=all $>0.99$ ) reliability for measuring joint angles. ${ }^{43}$

\section{CoP movement}

Additional secondary measures of standing balance will include CoP path length $(\mathrm{mm})$, anteroposterior and mediolateral excursion (range and SD, mm). Previous evidence in people with multiple sclerosis ${ }^{44}$ has demonstrated significant effects of wearing textured insoles within shoes, compared with wearing shoes only, including immediate reductions in mean CoP path length (the absolute length of CoP path movement) by $19.3 \%$ and sway rate (the mean speed of CoP movement) by $25.2 \%$, during quiet standing with eyes closed at baseline assessment.

\section{Patient-reported outcomes}

Patient-reported outcomes will include the Foot Health Status Questionnaire (FHSQ), a measure of self-perceived foot-health specific quality of life, before and after an intervention. ${ }^{45}$ The tool contains a total of 29 items (Likert scales and open-ended responses), of which 13 items address foot health, 6 items consider general health and 10 items address demographic and social factors. The tool has established content and criterion validity, ${ }^{46}$ as well as reliability. ${ }^{45}$ Further, the FHSQ has been shown to be responsive to change when measuring the effects of shoe insoles, ${ }^{47}$ and footwear interventions. ${ }^{48}$ Number of self-reported falls in the previous 12 months will also be recorded, and fear of falling assessed using the Falls Efficacy Scale-International (FES-I) ${ }^{49}$ The FES-I is a 16 -item tool which measures the level of concern experienced in relation to a range of activities of daily living. This tool has evidence for its convergent, predictive and internal validity and reliability, ${ }^{50}$ as well as responsiveness. ${ }^{52}$
Participants will also be followed for 4 weeks with insole wear self-reported diaries and falls calendars, ${ }^{53}$ to determine: (1) number of waking hours per day the insoles are worn and; (2) frequency, time, location of any falls/ injuries and what (if any) medical attention was sought. ${ }^{29}$

\section{Procedures}

Baseline assessment

On enrolment into the study, and following informed consent, participants will be mailed a series of questionnaires and a small wireless activity monitor (including instructions concerning the attachment and operation of the monitor). Demographics including sex, date of birth, height, weight, body mass index, medical history, current medications and diabetes duration will be collected, in addition to the patient-reported outcomes described above. Participants will be asked to wear the activity monitor for seven consecutive days, following which they will attend UQ for their baseline assessment. At this time, participants will return their completed questionnaires and activity monitor to the investigators. Balance, gait, foot sensation and proprioception will be assessed. To minimise the potential effects of fatigue, participants will be offered regular rests, and testing will be conducted at a similar time of day across the two sessions.

\section{Post intervention assessment}

Balance, gait, foot sensation, proprioception, self-reported foot health and fear of falling will be assessed within 2 weeks of the end of the 4-week intervention period, using the same procedures as at baseline. Physical activity will be monitored for a second time, over seven consecutive days, (ActivPAL mailed out at week 3, returned in person at the week 4 assessment). A 4-week intervention period is consistent with previous footwear intervention trials and will provide maximal time for the: (1) accrual of any sensorimotor training effects and (2) accumulation of meaningful changes in outcome measures related to balance performance. This final point of assessment will (1) quantify whether any immediate changes in balance and gait, observed at baseline, have accrued over time, or if additional effects can be seen and; (2) determine whether there are any alterations in physical activity levels, foot sensation or proprioception (which may suggest the insoles have a sensory training effect) or patient-reported outcomes. At the completion of the 4-week intervention, participants will be asked to rate the comfort of wearing their allocated insoles using a $100 \mathrm{~m}$ visual analogue scale anchored at $0 \mathrm{~mm}$ (extremely uncomfortable) and $100 \mathrm{~mm}$ (extremely comfortable). ${ }^{54}$ The perceived comfort rating scale has been previously validated and reported to be a reliable measures of footwear comfort. ${ }^{54}$ Participants will also be asked to return their insole-wear and falls diaries at this time.

\section{Statistical analysis}

Sample size was calculated for the primary outcome measure of CoP velocity, using data from Kalron et $a l^{44}$ 
who reported between-condition differences (shoes only and shoes + textured insole) that equated to an effect size of 0.72. Based on these data, 32 participants per group will provide sufficient power to detect differences in balance of a similar magnitude $(\alpha=0.05, \beta=0.20)$. We will allow for a $10 \%$ attrition rate over the 4 -week intervention period, and recruit 35 participants per group.

Demographic and clinical data will be described as mean (SD) for continuous data and n (\%) for categorical data. All analyses will be conducted in a blinded manner (by ALH, who will remain blinded to group allocation), on an intention-to-treat basis, with the level of significance set to 0.05 . At the end of the trial, a blind review of the data will be undertaken to consider the inclusion of covariates and the appropriateness of imputing any missing data. Where possible, reasons for missing data will be ascertained and reported (eg, voluntary withdrawal, loss to follow-up). All statistical analyses will be performed using SPSS V.24. Data will be examined for normality and homogeneity of variance. To establish any differences between the intervention and control groups for all outcome measures, a repeated measures mixed-models approach will be undertaken using data at baseline and 4 weeks. Non-parametric tests will be used where data are not normally distributed or violates the assumption of sphericity. A priori covariates will include age, gender, physical activity levels and insole wear time.

\section{Data management}

All data collected during the study will be stored securely (locked filing cabinets, password-protected computers) and confidentially (using a unique identification code) on paper and electronically at UQ, and will only be accessible to the investigators. Following the study, data will be stored for at least 10 years.

\section{Patient and public involvement}

In our previous studies of textured shoe insoles, we received feedback from patients about their experiences and perceptions of wearing our insoles and completing the assessment procedures, which guided the design of the current study and development of the intervention. Patients were not involved in the development of the research question, outcome measures or the recruitment of participants. We will assess the burden of the insole intervention on patients by collecting information about adverse events and the number of hours the insoles were worn. The results of the study will be disseminated to patients via media releases through Diabetes Australia (and its state-specific organisations, eg, Diabetes Queensland).

\section{Ethics and dissemination}

Written informed consent will be obtained from all participants prior to enrolment.

There are minimal risks associated with this study. The textured and smooth insoles will be constructed from materials used in standard insoles traditionally issued to people with diabetic peripheral neuropathy. The allocated insole will not be fitted if it is markedly uncomfortable for the participant to wear, to minimise any adverse reactions.

Exposure to a new insole could lead to dermatological, musculoskeletal or sensory changes to the feet, or issues related to insole-fit and durability. Subsequently, all participants will receive contact details for podiatry care, and an insole advice leaflet. Participants who develop adverse foot events (eg, ulceration) will be withdrawn from the trial. Similarly, should wearing insoles increase the prevalence of falls experienced during the intervention period (relative to the frequency of falls experienced in the previous 12 months), all participants to whom this concerns will be withdrawn from the study, and their general practitioner notified. All adverse events will be reported in line with university ethical governance standards.

During balance and walking tests, while wearing unfamiliar shoes and insoles, participants could experience a change in their stability. Therefore, participants will be closely supervised by the investigators. As part of the screening assessment, participants will be required to report on personal pain, disease and injury. This may require disclosure of the cause of impairment, or recollection of traumatic events (eg, injurious fall), which could be a potential cause of distress. Participants will be advised that they can choose not to answer any questionnaire items, and that this decision will not affect their involvement in the trial.

The findings will be disseminated at national and international conferences and through peer-reviewed journals. Patients, healthcare professionals and the public will be informed of the findings via workshops, local meetings and social media.

\section{Author affiliations}

${ }^{1}$ School of Health and Rehabilitation Sciences, University of Queensland, Brisbane, Queensland, Australia

${ }^{2}$ School of Human Movement and Nutrition Sciences, University of Queensland, Brisbane, Queensland, Australia

${ }^{3}$ Faculty of Health Sciences, The University of Sydney, Sydney, New South Wales, Australia

${ }^{4}$ Peninsula Clinical Trials Unit, University of Plymouth, Plymouth, UK

${ }^{5}$ Institute of Health and Biomedical Innovation, Queensland University of Technology

- Kelvin Grove Campus, Brisbane, Queensland, Australia

${ }^{6}$ Health and Rehabilitation Research Institute, Auckland University of Technology, Auckland, New Zealand

Contributors ALH led the conception, design and writing of the study protocol with substantial contributions to the design, writing, critical review of intellectual content and final manuscript approval from EMG, JNM, JB, JP, GK and KR. All authors agree to be accountable for their work. As principal investigator, ALH takes overall responsibility for the work. EMG made substantial contributions (as the first research assistant) to participant recruitment and data collection strategies in the development and initial stages of the study and protocol. JNM (as the study podiatrist), provided specific expertise in the development and construction of the textured and smooth insoles, dispensing the insoles and providing clinical support to participants over the intervention period. JB, JP, KR and GK made substantial contributions to the trial design and statistical analysis strategy. JP and KR were also responsible for providing expertise concerning the clinical management of diabetic feet. GK provided expertise in all biomechanical testing procedures and outcome measures. 
Funding This work is supported by a 2017 Diabetes Australia Research Program General Grant (Y17G-HATA), awarded to ALH, JB, JP, GK and KR.

Competing interests Materials used to construct the textured and smooth insoles investigated in this study were supplied by Algeos PTY Ltd. (VIC, Australia). The company had no involvement in the conception or design of the study or preparation of this manuscript; and will not be involved in subsequent data acquisition, analysis or interpretation.

Patient consent for publication Not required.

Ethics approval The study has been approved by the UQ Human Research Ethics Committee (\#2017000098).

Provenance and peer review Not commissioned; externally peer reviewed.

Open access This is an open access article distributed in accordance with the Creative Commons Attribution Non Commercial (CC BY-NC 4.0) license, which permits others to distribute, remix, adapt, build upon this work non-commercially, and license their derivative works on different terms, provided the original work is properly cited, appropriate credit is given, any changes made indicated, and the use is non-commercial. See: http://creativecommons.org/licenses/by-nc/4.0/.

\section{REFERENCES}

1. Jaiswal M, Divers J, Dabelea D, et al. Prevalence of and Risk Factors for Diabetic Peripheral Neuropathy in Youth With Type 1 and Type 2 Diabetes: SEARCH for Diabetes in Youth Study. Diabetes Care 2017;40:1226-32.

2. Van Acker K, Bouhassira D, De Bacquer D, et al. Prevalence and impact on quality of life of peripheral neuropathy with or without neuropathic pain in type 1 and type 2 diabetic patients attending hospital outpatients clinics. Diabetes/metabolism research and reviews 2009;35:206-13.

3. Macgilchrist C, Paul L, Ellis BM, et al. Lower-limb risk factors for falls in people with diabetes mellitus. Diabet Med 2010;27:162-8.

4. Brown SJ, Handsaker JC, Bowling FL, et al. Diabetic peripheral neuropathy compromises balance during daily activities. Diabetes Care 2015;38:1116-22.

5. Ducic I, Short KW, Dellon AL. Relationship between loss of pedal sensibility, balance, and falls in patients with peripheral neuropathy. Ann Plast Surg 2004;52:535-40.

6. Richardson JK, Hurvitz EA. Peripheral neuropathy: a true risk factor for falls. J Gerontol A Biol Sci Med Sci 1995;50:M211-5.

7. Lavery LA, LaFontaine J, Higgins KR, et al. Shear-reducing insoles to prevent foot ulceration in high-risk diabetic patients. Adv Skin Wound Care 2012;25:519-24.

8. Paton JS, Stenhouse EA, Bruce G, et al. A comparison of customised and prefabricated insoles to reduce risk factors for neuropathic diabetic foot ulceration: a participant-blinded randomised controlled trial. J Foot Ankle Res 2012;5:31.

9. Ites $\mathrm{KI}$, Anderson EJ, Cahill ML, et al. Balance interventions for diabetic peripheral neuropathy: a systematic review. J Geriatr Phys Ther 2011;34:109-16.

10. Priplata AA, Patritti BL, Niemi JB, et al. Noise-enhanced balance control in patients with diabetes and patients with stroke. Ann Neurol 2006;59:4-12.

11. Van Geffen JA, Dijkstra PU, Hof AL, et al. Effect of flat insoles with different Shore A values on posture stability in diabetic neuropathy. Prosthet Orthot Int 2007;31:228-35.

12. Singleton JR, Marcus RL, Lessard MK, et al. Supervised exercise improves cutaneous reinnervation capacity in metabolic syndrome patients. Ann Neurol 2015;77:146-53.

13. Hatton AL, Dixon J, Rome K, et al. Standing on textured surfaces: effects on standing balance in healthy older adults. Age Ageing 2011;40:363-8.

14. Hatton AL, Dixon J, Rome K, et al. Altering gait by way of stimulation of the plantar surface of the foot: the immediate effect of wearing textured insoles in older fallers. J Foot Ankle Res 2012;5:11.

15. Orth D, Davids K, Wheat J, et al. The role of textured material in supporting perceptual-motor functions. PLoS One 2013;8:e60349.

16. Qiu F, Cole MH, Davids KW, et al. Effects of textured insoles on balance in people with Parkinson's disease. PLoS One 2013;8:e83309.

17. Maki BE, Holliday PJ, Topper AK. A prospective study of postural balance and risk of falling in an ambulatory and independent elderly population. J Gerontol 1994;49:M72-84.

18. Stel VS, Smit JH, Pluijm SM, et al. Balance and mobility performance as treatable risk factors for recurrent falling in older persons. J Clin Epidemiol 2003;56:659-68.
19. Dixon J, Hatton AL, Robinson J, et al. Effect of textured insoles on balance and gait in people with multiple sclerosis: an exploratory trial. Physiotherapy 2014;100:142-9.

20. Paton J, Hatton AL, Rome K, et al. Effects of foot and ankle devices on balance, gait and falls in adults with sensory perception loss: a systematic review. JBI Database System Rev Implement Rep 2016;14:127-62.

21. Paton J, Glasser S, Collings R, et al. Getting the right balance: insole design alters the static balance of people with diabetes and neuropathy. J Foot Ankle Res 2016;9:40.

22. Rao N, Aruin AS. Auxiliary sensory cues improve automatic postural responses in individuals with diabetic neuropathy. Neurorehabil Neural Repair 2011;25:110-7.

23. Wegener C, Wegener K, Smith R, et al. Biomechanical effects of sensorimotor orthoses in adults with Charcot-Marie-Tooth disease. Prosthet Orthot Int 2016;40:436-46.

24. Chan AW, Tetzlaff JM, Gøtzsche PC, et al. SPIRIT 2013 explanation and elaboration: guidance for protocols of clinical trials. BMJ 2013;346:e7586.

25. Meijer JW, Smit AJ, Sonderen EV, et al. Symptom scoring systems to diagnose distal polyneuropathy in diabetes: the Diabetic Neuropathy Symptom score. Diabet Med 2002;19:962-5.

26. The Royal Australian College of General Practitioners. General practice management of type 2 diabetes: 2016-18. East Melbourne, Vic: RACGP, 2016.

27. Folstein MF, Folstein SE, McHugh PR, state" "Mini-mental. "Minimental state". A practical method for grading the cognitive state of patients for the clinician. J Psychiatr Res 1975;12:189-98.

28. Fernando M, Crowther R, Lazzarini P, et al. Biomechanica characteristics of peripheral diabetic neuropathy: A systematic review and meta-analysis of findings from the gait cycle, muscle activity and dynamic barefoot plantar pressure. Clin Biomech 2013;28:831-45.

29. Hatton AL, Dixon J, Rome K, et al. The effects of prolonged wear of textured shoe insoles on gait, foot sensation and proprioception in people with multiple sclerosis: study protocol for a randomised controlled trial. Trials 2016;17:208.

30. Raymakers JA, Samson MM, Verhaar HJ. The assessment of body sway and the choice of the stability parameter(s). Gait Posture 2005;21:48-58

31. Mcllroy WE, Maki BE. Preferred placement of the feet during quiet stance: development of a standardized foot placement for balance testing. Clin Biomech 1997;12:66-70.

32. Batey P, Rome K, Finn P, et al. Assessing Reliability of Measurement of Gait Velocity. Physiotherapy 2003;89:313-7.

33. Menz HB, Latt MD, Tiedemann A, et al. Reliability of the GAITRite walkway system for the quantification of temporo-spatial parameters of gait in young and older people. Gait Posture 2004;20:20-5.

34. Dahlgren G, Carlsson D, Moorhead A, et al. Test-retest reliability of step counts with the ActivPAL ${ }^{\mathrm{TM}}$ device in common daily activities. Gait Posture 2010;32:386-90.

35. Grant PM, Ryan CG, Tigbe WW, et al. The validation of a novel activity monitor in the measurement of posture and motion during everyday activities. Br J Sports Med 2006;40:992-7.

36. Ryan CG, Grant PM, Tigbe WW, et al. The validity and reliability of a novel activity monitor as a measure of walking. Br J Sports Med 2006;40:779-84.

37. Loprinzi PD, Hager KK, Ramulu PY. Physical activity, glycemic control, and diabetic peripheral neuropathy: a national sample. $J$ Diabetes Complications 2014;28:17-21.

38. Najafi B, Armstrong DG, Mohler J. Novel wearable technology for assessing spontaneous daily physical activity and risk of falling in older adults with diabetes. J Diabetes Sci Technol 2013;7:1147-60.

39. Armstrong DG, Lavery LA, Vela SA, et al. Choosing a practical screening instrument to identify patients at risk for diabetic foot ulceration. Arch Intern Med 1998;158:289-92.

40. Citaker S, Gunduz AG, Guclu MB, et al. Relationship between foot sensation and standing balance in patients with multiple sclerosis. Gait Posture 2011;34:275-8.

41. Richard JL, Reilhes L, Buvry S, et al. Screening patients at risk for diabetic foot ulceration: a comparison between measurement of vibration perception threshold and 10-g monofilament test. Int Wound J 2014;11:147-51.

42. Riskowski JL, Mikesky AE, Bahamonde RE, et al. Proprioception, gait kinematics, and rate of loading during walking: are they related? J Musculoskelet Neuronal Interact 2005;5:379-87.

43. Russell TG, Jull GA, Wootton R. Can the Internet be used as a medium to evaluate knee angle? Man Ther 2003;8:242-6.

44. Kalron A, Pasitselsky D, Greenberg-Abrahami M, et al. Do textured insoles affect postural control and spatiotemporal parameters of 
gait and plantar sensation in people with multiple sclerosis? $\mathrm{Pm} R$ 2015;7:17-25.

45. Bennett PJ, Patterson C, Wearing S, et al. Development and validation of a questionnaire designed to measure foot-health status. J Am Podiatr Med Assoc 1998;88:419-28.

46. Cuesta-Vargas Al, Galan-Mercant A, Martín-Borras MC, et al. Criterion-related validity of the foot health status questionnaire regarding strength and plantar pressure measurements in elderly people. Foot Ankle Spec 2012;5:366-73.

47. Landorf KB, Keenan AM. An evaluation of two foot-specific, health-related quality-of-life measuring instruments. Foot Ankle Int 2002;23:538-46.

48. Menz HB, Auhl M, Ristevski S, et al. Comparison of the responsiveness of the foot health status questionnaire and the Manchester foot pain and disability index in older people. Health Qual Life Outcomes 2014;12:158.

49. Yardley L, Beyer N, Hauer K, et al. Development and initial validation of the Falls Efficacy Scale-International (FES-I). Age Ageing 2005;34:614-9.
50. Delbaere K, Close JC, Mikolaizak AS, et al. The Falls Efficacy Scale International (FES-I). A comprehensive longitudinal validation study. Age Ageing 2010;39:210-6.

51. Hill H, McMeekin P, Parry SW. Does the falls efficacy scale international version measure fear of falling: a reassessment of internal validity using a factor analytic approach. Age Ageing 2014;43:559-62.

52. Petrella RJ, Payne M, Myers A, et al. Physical function and fear of falling after hip fracture rehabilitation in the elderly. Am J Phys Med Rehabil 2000;79:154-60.

53. Spink MJ, Menz HB, Fotoohabadi MR, et al. Effectiveness of a multifaceted podiatry intervention to prevent falls in community dwelling older people with disabling foot pain: randomised controlled trial. BMJ 2011;342:d3411.

54. Mills $\mathrm{K}$, Blanch $\mathrm{P}$, Vicenzino $\mathrm{B}$. Identifying clinically meaningful tools for measuring comfort perception of footwear. Med Sci Sports Exerc 2010;42:1966-71. 